TABLE III

Average annual total costs of reprovision compared to the costs of care in Darenth Park (1987-88 prices $\mathrm{Es)}$

\begin{tabular}{lcccc}
\hline & Recurrent & $\begin{array}{c}\text { Personal } \\
\text { consumption }\end{array}$ & Capital & Total \\
\hline NHS hostels and centres & 16,066 & 715 & 3,500 & 20,281 \\
Group homes & 24,808 & 757 & 4,271 & 29,836 \\
$\begin{array}{l}\text { Total (weighted av.) } \\
\text { Average annual costs } \\
\text { of hospital care at }\end{array}$ & 20,715 & 740 & 3,800 & 25,225 \\
\begin{tabular}{l} 
Darenth Park \\
\hline
\end{tabular} & 12,452 & 429 & 3,500 & 16,500 \\
\hline
\end{tabular}

vided in old hospitals where staff were very difficult to recruit, it should come as no surprise that modern facilities and better staffing cost more money. The strange thing is that anyone ever believed otherwise.

These findings should be of particular importance to planners and practitioners in the light of the Government's proposals to change the basis of funding for community care anmounced in the recent White Paper (DoH, 1989). Social security funds will no longer be available to meet the costs of care in the community. Given the important part we have demonstrated it has played in reprovision, it will be critical to future plans that adequate alternative allocations are made to the local or health authorities seeking to make this policy a humane reality.

\section{References}

Department of Health Cmd 849 Caring for People: Community Care in the Next Decade and Beyond. London: HMSO.

KNaPP, M. (1987) The Cost Effectiveness of Psychiatric Reprovision Services. Discussion paper No. 533/2. Canterbury, PSSRU, University of Kent.

KORMAN, N. \& GlenNERster, H. (1990) Hospital Closure: A Political and Economic Study. Milton Keynes: Open University Press.

SHEILL, A. \& WRIGHT, K. (1988) Counting the Cost of Community Care: York University Centre for Health Economics.

WING, L. (1989) Hospital Closure and the Re-settlement of Residents. Aldershot: Gower.

\title{
Cross-cultural psychiatric research: an anthropologist's view
}

\section{INGA-BRITT KRAUSE, Social Anthropologist and Researcher, Academic Department of Psychiatry, University College and Middlesex School of Medicine, Wolfson Building, The Middlesex Hospital, London W1N 8AA}

A need for improved communication between the social sciences and psychiatry is being expressed from many quarters. Interest in social and cultural issues is not, of course, new to psychiatry, but collaboration between the two approaches has not always been easy. Recently one social science in particular has become popular with psychiatry. This is social anthropology, and many psychiatrists consider that the inclusion of anthropological data and methods, particularly in cross-cultural research, can be useful and informative to psychiatry. What then is the relationship between anthropology and psychiatry and what are the problems which professionals face in attempting interdisciplinary research? 


\section{Anthropology and psychiatry}

Social anthropology is distinguished from other branches of the social sciences by its history and its emphasis, if not necessarily by the methods employed by every social anthropologist. Two strategies typify anthropological research, the first is a strength and the second may be a weakness. Firstly, anthropological analysis begins from the premise that, regardless of the problem to be researched, no aspect of a society or a culture is excluded $a$ priori from the enquiry. Anthropological research begins, not with a problem, but with a particular social, cultural and historical context. Within this context the initial problem may require drastic reformulation, but that is a later stage. Secondly, anthropological research emphasises qualitative data. This includes data from different aspects of a culture or a society such as data on language, ideology, kinship structure, economic and political structure, myths, classification, notions of the 'self', notions of 'the person', ideas about illness etc. Often the connection between these different areas of data is not clear when the data are collected and sometimes data which seem central at the outset, turn out to be relatively unimportant. The collection of such diverse types of qualitative data is emphasised to the almost total exclusion of statistical data. Indeed, social anthropologists tend to be more unfamiliar with statistical methods than any other type of social scientist.

The approach of social anthropology is therefore very different from that of psychiatry. Anthropology focuses on context, on cultural notions, on the meaning of symbols and social structural arrangements, whereas psychiatry, to the extent that it is derived from the medical model, is problem-orientated and preoccupied with pathology. Perhaps these differences account for the increasing popularity of social anthropology with psychiatrists in recent years. For their own part, many anthropologists have welcomed the opportunity to consider their material within a broader theoretical framework and the challenge to put this material to use in the specific context of the clinical encounter.

The initial communication between the two disciplines, then, tends to begin in a spirit of enthusiasm and with great promise. When two such different disciplines get together to examine the context, causes and expressions of human suffering something valuable surely will emerge. However, difficulties soon arise. Consider the following dialogue between a psychiatrist and an anthropologist who are setting out to collaborate.

Psychiatrist I have this problem with those of my patients who come from social and cultural backgrounds which are unfamiliar to me. I do not know anything about their language and their culture. They seem to talk a lot about their bodies, but I feel unsure about making diagnoses. We have a very good paradigm for understanding mental suffering, but I need to be able to translate the ideas these people present to me into my psychiatric categories to treat them successfully. You are an anthropologist, can you tell me something about these people's culture and the way they think about mental illness? Anthropologist I think I can help you. But first let me tell you this about culture: different cultural concepts, patterns of behaviour and modes of thought can be immensely varied and they may have influences on the body and the mind which we do not recognise. But if you give me a year, I will find out about these patients for you.

Psychiatrist Good God, a whole year. What will you be doing in all that time?

Anthropologist Well, first I will brush up on my skill in speaking the language these people speak. Then I will begin by hanging around on street corners and in people's homes. That will give me some pretty good ideas about how they think about illness and mental problems. Then I can start working on your problems. Psychiatrist But surely you know a lot about this culture already. You have worked with people from this part of the world all your life and there must be a lot of books available.

Anthropologist Sure, books and my previous work are useful. But $I$ also need to know about these people in this specific context. I cannot be sure how they think.

Psychiatrist Well, you know my real problem with culture and cultural ideas is that I can't measure these aspects. What instruments are you going to use? Anthropologist By the end of the year I expect I will be able to tell you a lot about the aspects of this culture and the way these people think which you find troublesome - but I can't measure these things, I do not think that that is possible. Certainly not at such an early stage.

Perhaps these two people will be able to work something out but there are many problems and our two professionals may not be friends by the end of the collaboration. Probably the focus for disagreement will be the translation between emic and etic categories, that is to say between particular and universal aspects of illness. Anthropologists have always been uncomfortable with etic categories particularly when they have made it their business to examine hermeneutic or the 'meaning' aspects of human existence. However, it is also in this area of meaning where anthropology can make the most significant contribution to the study of illness. Psychiatrists have been similarly uncomfortable with emic material. This tension in the area of translation is clearly shown in the debate about culture-bound syndromes but it also surfaces in other areas of cross-cultural psychiatry. 


\section{Problems with translation between emic and etic \\ Language}

There are three specific areas of cross-cultural psychiatric research in which our team is likely to encounter problems. First, there are problems posed by language differences. The use of questionnaires and symptom inventories is commonplace in crosscultural psychiatric research and usually such questionnaires contain lists of items or symptoms derived from Western psychiatric nosology. The standard procedure is to translate the items into the appropriate language, to backtranslate and finally to assess the backtranslated version against the original. Although many non-Western languages do not have terms which denote standard psychiatric categories such as depression, at this technical level it is often possible with rewording and the use of different idioms to arrive at an acceptable translation of particular items or symptoms. In translating the GHQ into Punjabi we found relatively few problems. It was necessary to use a significantly different wording for only two questions. One of these was the question "Have you been feeling run down and out of sorts?" in the English version. In the Punjabi version this reads "Do you feel that your heart is sinking and feel different?". This translation was acceptable both to the bilingual Punjabis who helped us translate the GHQ and to the bilingual psychologist who checked it for us and we therefore have no reason to doubt its technical validity.

However, careful and technically correct translations may contain problems because such a procedure does not include any attempt at interpretation of items or consideration of their cultural contexts. The "sinking heart" illustrates this well. In Punjabi the notion of "sinking heart" conveys feelings of general weakness, but it also refers to a wider and in some way more concrete set of sensations and emotional states including physical sensations in the chest, sadness, worry, anxiety caused by physical exhaustion or social and personal failure (Krause, 1989). The expression is used by individuals in various contexts. Healthy people may use it to refer to the normal sufferings and anxieties they experience during the course of their lives. Mentally ill patients may use it to denote despair and depression and patients suffering from some form of heart disease may use it to refer to their heart symptoms. The English version "to feel run down and out of sorts", although technically correct, does not convey this complexity. There is only broad agreement between the item in the two languages. In both, the item refers to a somatic complaint (physical weakness) and its inclusion in the somatic subscale of both the English and the Punjabi version of the GHQ-28 is appropriate. However, the Punjabi item could equally well be included in the depressive subscale and it may even be argued that "sinking heart" is a symptom of social dysfunction.

\section{Conceptual equivalence}

This points to the second major area of problems in cross-cultural psychiatric research. Technically equivalent translations may not be conceptually equivalent. Whether indeed they are conceptually acceptable can only be demonstrated by research aimed at understanding the meaning of the sensations and emotions to which the item on the questionnaire refers. This involves a careful consideration of the cultural context and this is where cross-cultural psychiatric research tends to stumble and fail.

The distinction so often drawn between somatic and psychological symptoms is a case in point. One explanation put forward to account for somatisation in non-Western patient populations is that these cultures lack an extensive emotional vocabulary. By contrast, in Western cultures patients are able to present psychological symptoms more accurately (Leff, 1988). Careful analysis of the data does not support this. In the first place, many non-Western languages have nuances and rich emotional vocabularies. Secondly, even if a complaint is expressed in somatic terms, these terms may themselves also denote psychological and emotional states. This is for example the case with "semen-loss" and the "sinking heart" referred to above. Both of these derive from cultural contexts in which the relationship between mind and body are conceived differently than in Western contexts. Thirdly, recent psychiatric research with British and American patients also suggests that somatisation is a worldwide phenomenon (Lipowski, 1988). In all these respects, the differences between Western and nonWestern cultures is not startling and the somatic/ psychological distinction has been unhelpful insofar as it has steered research away from other cultural differences (Littlewood, 1990).

Anthropological work points to other and perhaps more pertinent differences between cultures. In many non-Western cultures a sharp distinction between body and mind is not drawn, and there is also cultural variation in the perception, classification and evaluation of emotions and in the emphasis and meaning placed on parts of the human body. All these issues pertain to the emic domain and are related to differences in philosophical and cultural orientation and they are all relevant to the investigation of mental illness cross-culturally.

\section{Anthropological validation}

The acknowledgement of conceptual differences presents cross-cultural psychiatric research with a third problem. This is the problem of validation and 
of how practically to carry out sound cross-cultural research. The procedure for psychiatric validation in non-Western settings follows that pioneered by Goldberg (Goldberg, 1972) and is now also used widely in the application of new questionnaires in Western settings. The scores obtained from questionnaires are assessed against the judgement obtained from other standard psychiatric instruments such as the PSE, BPI and the DSM-III. Although results obtained in this manner are generally accepted by psychiatrists, it is doubtful whether this procedure adequately addresses the problems under investigation. The underlying assumption is that mental distress is fundamentally similar, if not in presentation, at least in underlying pathology across different cultures and therefore that Western psychiatric nosology can be applied as a yardstick. Some psychiatrists have been duly sceptical about this and have added lists of indigenous symptoms and syndromes to the questionnaires. However, even in such carefully conducted and culturally sensitive studies, criteria from Western diagnostic taxonomies are usually used for validation and there have been very few attempts at validation with reference to the meaning attributed to symptoms within particular cultural contexts.

Arthur Kleinman has drawn attention to this and suggested that most standard psychiatric crosscultural research commits "category fallacies" (Kleinman, 1987). In Kleinman's words, a "category fallacy" is "the reification of a nosological category developed for a particular cultural group that is then applied to members of another culture for whom it lacks coherence and its validity is not established" (p. 452). Anthropological validation includes a contextual and ethnographic approach to the translation of symptoms and syndromes and anthropological data therefore offer a test of whether Western categories, upon which research and diagnostic instruments are based, have coherence and are valid for the particular culture and population in which research is being carried out. Without this anthropological validation the result may be a "category fallacy", that is to say a partial and perhaps even tautological picture of the cross-cultural incidence and prevalence of mental illness.

\section{Concluding remarks}

Let me return to our research team and consider how the problems which exist in the translation from one cultural context to another may affect their collaboration. Although this is not always an issue in anthropological research, the anthropologist who ventures into cross-cultural medical research must accept that the issue of translation is a necessary and pragmatic aim. The anthropological approach of collecting, analysing in depth and interpreting ethnographic and contextual material is well suited to this aim and sound psychiatric research, particularly when it addresses cross-cultural issues, needs data produced in this manner. However, in research psychiatrists also find this qualitative, contextual and philosophical data problematic and difficult to integrate with standard psychiatric research models. This is because anthropological data may indicate that particular cultures do not have valid constructs which fit Western notions and consequently the qualitative data produced by the anthropologist may challenge the very bricks of Western medical nosology.

This makes for an uncomfortable relationship between our two professionals. If, as is often the case in cross-cultural psychiatric research, anthropological data are used selectively and in a superficial manner, anthropology is compromised and the value of the anthropological approach, both theoretically and methodologically, is lost to psychiatry. To be useful in research collaboration, the anthropologist must be allowed to explore specific cultural settings and constructs in depth and the relationship between the two disciplines must be one of equality. Sound cross-cultural psychiatric research should therefore always involve collaborations between anthropologists and psychiatrists and produce both qualitative and quantitative data.

This has practical implications for the planning of research projects. It is, for example, necessary to set aside adequate time and resources for the exploration of anthropological themes in the initial phases of research. Rather than beginning with the use of Western diagnostic instruments in different cultural settings, sound cross-cultural psychiatric research aims at the development of concepts and possibly instruments which are culturally sensitive and therefore properly comparative.

\section{References}

GOLDBERG, D. P. (1972) The Detection of Psychiatric Illness by Questionnaire. Oxford University Press.

KLeINMAN, A. (1987) Anthropology and psychiatry; the role of culture in cross-cultural research on illness. British Journal of Psychiatry, 151, 447-454.

KraUSE, I-B. (1989) Sinking heart: a Punjabi communication of distress. Social Science and Medicine, 29, 563-575.

LEFF, J. (1988) Psychiatry around the Globe: A Transcultural View. Second edition. London: Royal College of Psychiatrists.

LIPOWSKı, Z. J. (1988) Somatization: The concept and its clinical application. American Journal of Psychiatry, 145, 1358-1368.

LITTLEWOOD, R. (1990) From categories to context: A decade of the "new cross-cultural psychiatry". British Journal of Psychiatry, 156, 308-327.

A full list of references is available from the author. 